Supporting Information for:

\title{
The Effects of Alkylammonium Choice on the Stability and Performance of Quasi-2D Organolead Halide Perovskites
}

\author{
Bryan R. Wygant, ${ }^{a}$ Alexandre Z. Ye ${ }^{b}$ Andrei Dolocan, ${ }^{c}$ and C. Buddie Mullins ${ }^{a, b, c, *}$ \\ ${ }^{a}$ Department of Chemistry, ${ }^{b}$ McKetta Department of Chemical Engineering, ${ }^{c}$ Texas Material Institute; University of \\ Texas at Austin, Austin, TX 78712 \\ *corresponding author: mullins@che.utexas.edu, (512)-471-5817
}



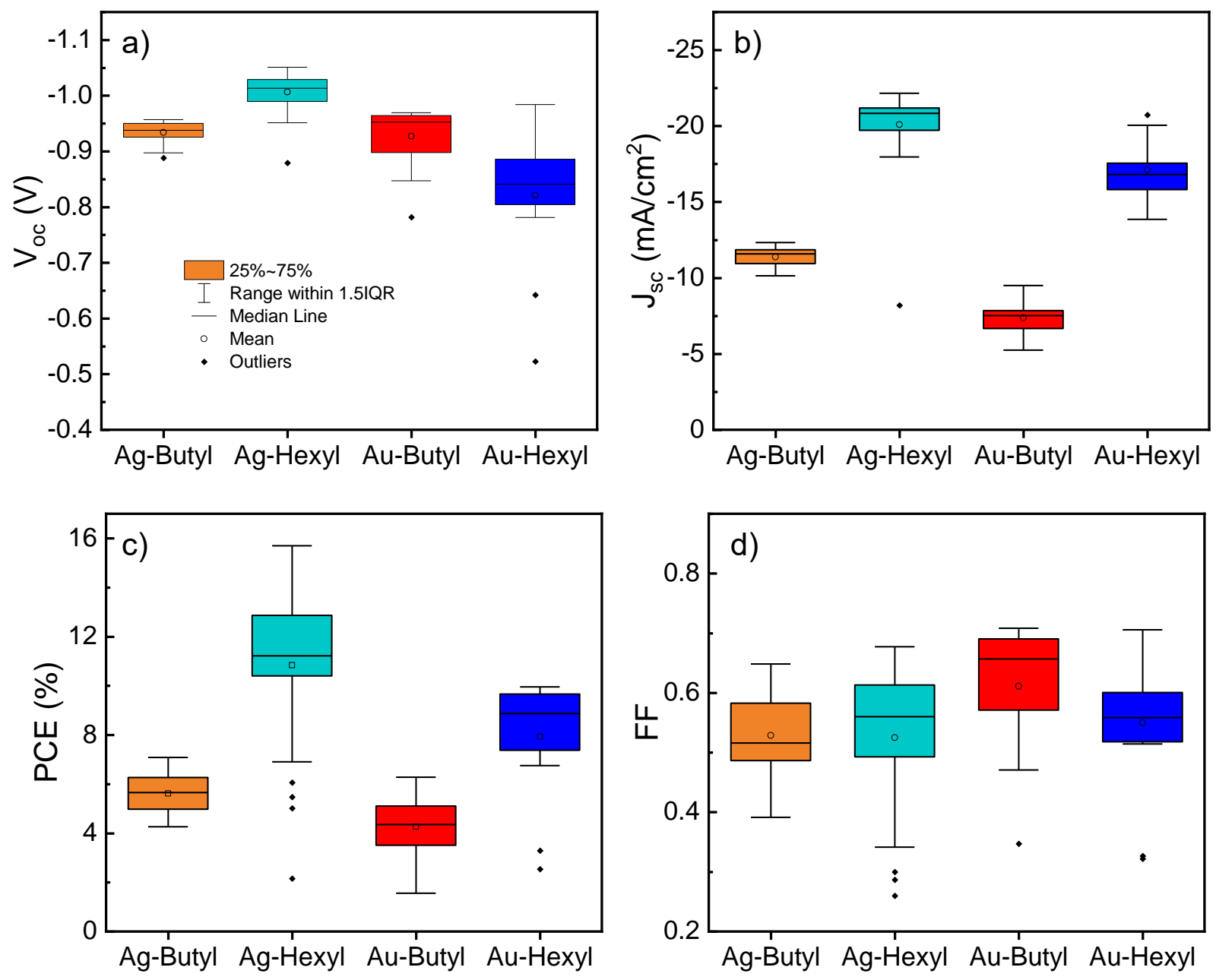

Figure S1 Average (a) $\mathrm{V}_{o c}$, (b) $\mathrm{J}_{\mathrm{sc}}$, (c) PCE, and (d) FF of all butyl- and hexyl-MAPI devices with both Ag and Au contacts. 

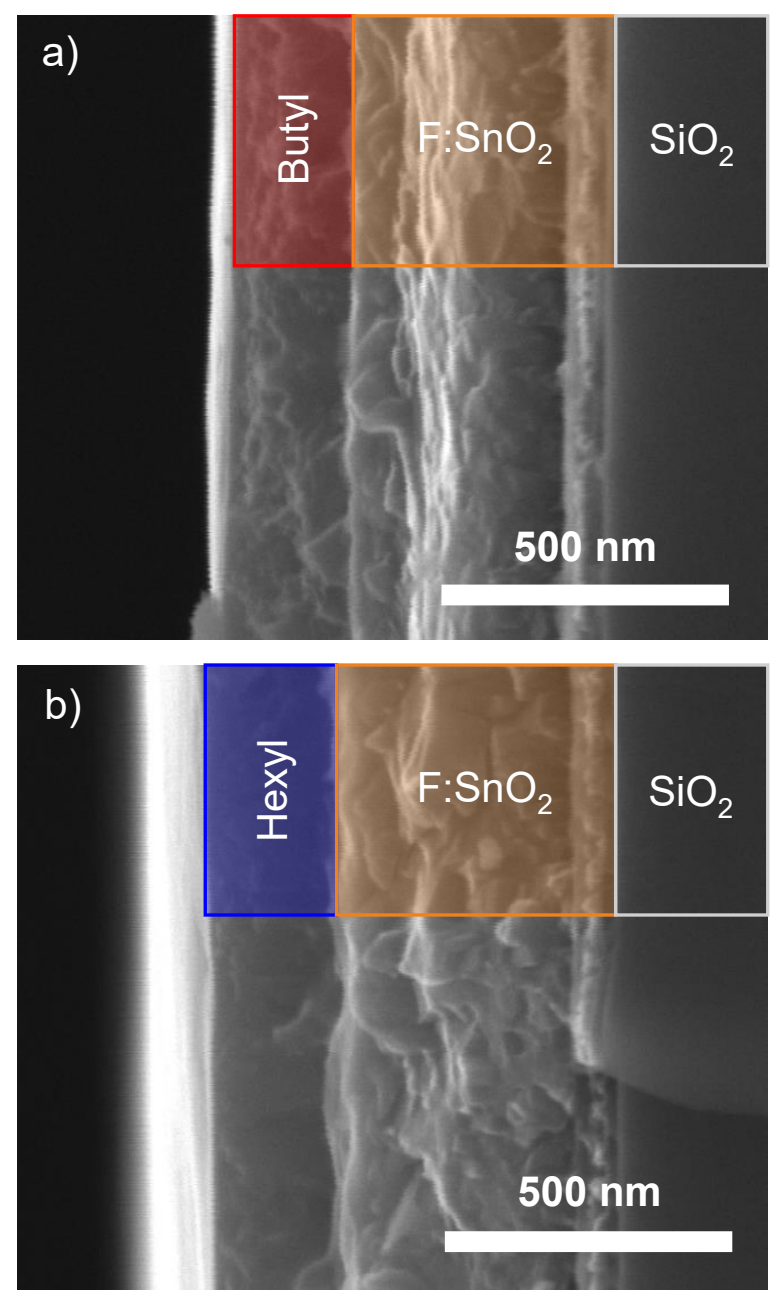

Figure S2 Cross sectional SEM image of (a) butylMAPI and (b) hexyl-MAPI films. 

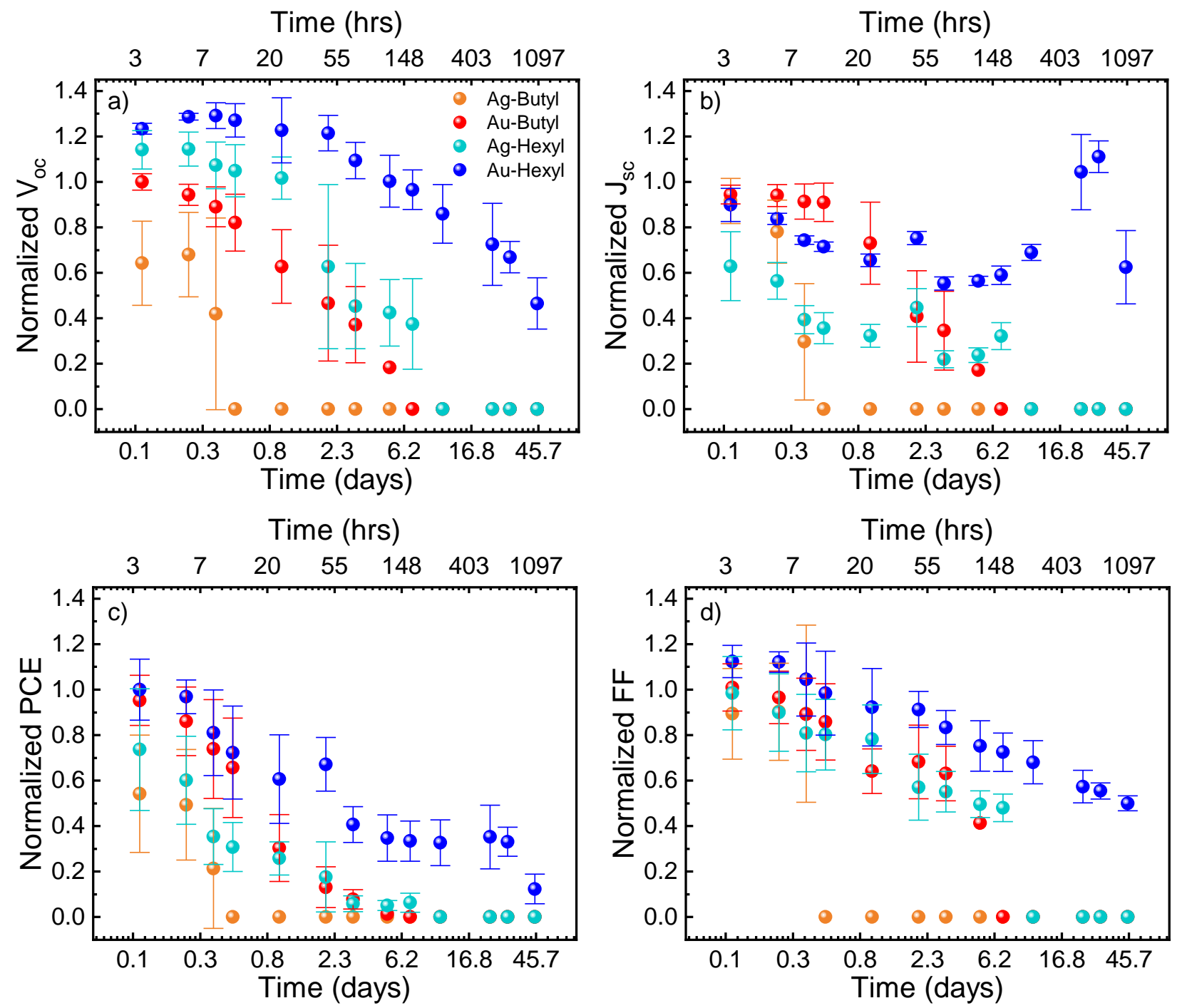

Figure $\mathbf{S 3}$ Normalized (a) $\mathrm{V}_{\text {oc }}$, (b) $\mathrm{J}_{\mathrm{sc}}$, (c) PCE, and (d) FF of butyl- and hexyl-MAPI devices fabricated using Au and $\mathrm{Ag}$ contacts exposed to $78 \% \mathrm{RH}$. 

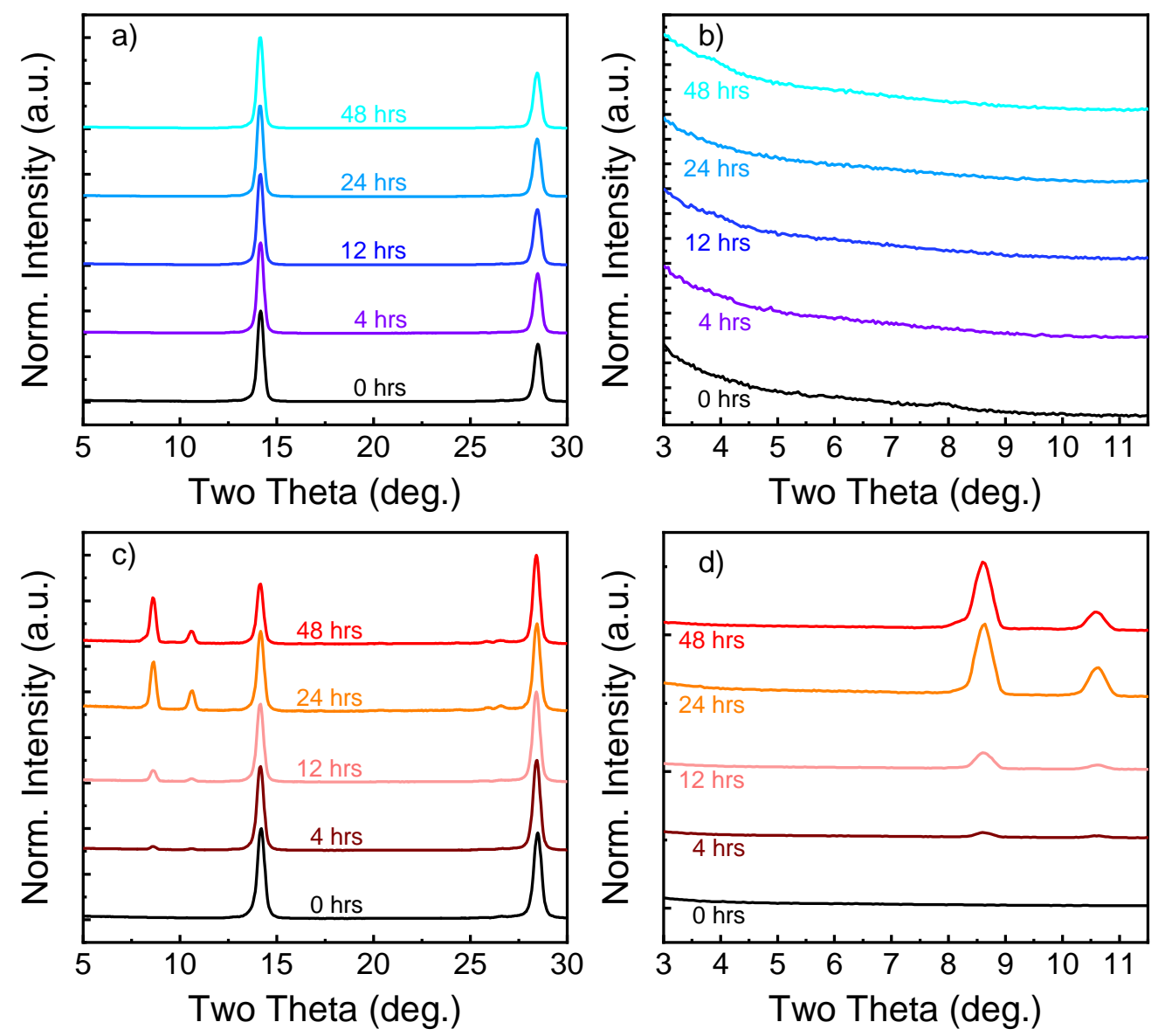

Figure S4 Full XRD spectra of (a) hexyl-MAPI and (c) butyl-MAPI films exposed to 78\%RH over 48 hours. Low-angle XRD spectra of (b) hexyl-MAPI and (d) butyl-MAPI films exposed to humidity. As exposure time progresses, the butyl-MAPI films exhibit the growth of low- $n$ peaks below $2 \theta=12^{\circ}$, while hexyl-MAPI does not. 

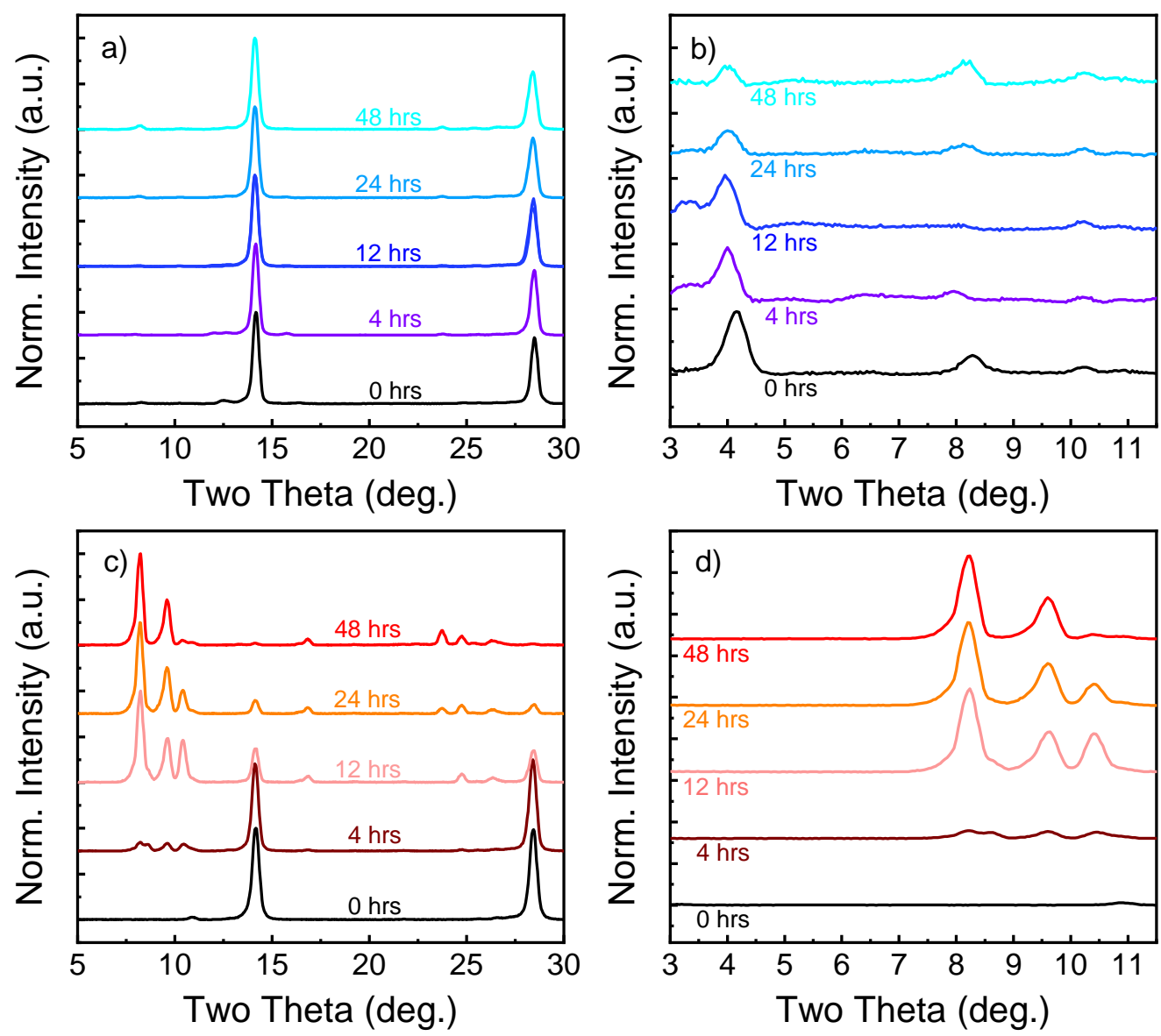

Figure S5 Full XRD spectra of (a) Ag-hexyl and (c) Ag-butyl devices exposed to 78\%RH over 48 hours. Low-angle XRD spectra of (b) Ag-hexyl and (d) Ag-butyl devices exposed to humidity. As exposure time progresses, the Ag-butyl devices exhibit the growth of low- $n$ peaks below $2 \theta=12^{\circ}$ and the near complete loss of the original diffraction peaks, while hexyl-MAPI does not. Weak low- $n$ peaks are visible in (b), likely indicating the formation of some low- $n$ species during fabrication. 

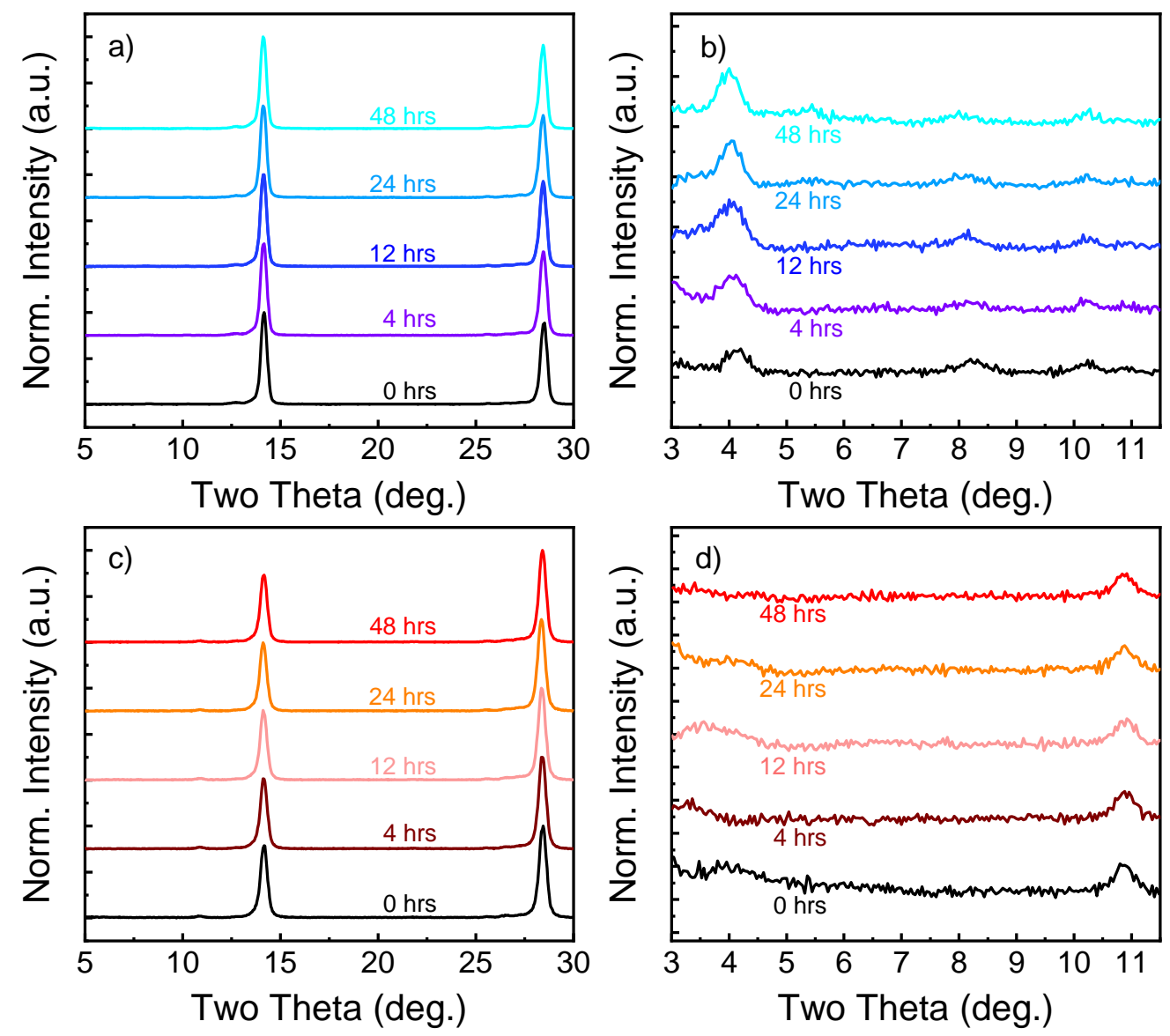

Figure S6 Full XRD spectra of (a) Au-hexyl and (c) Au-butyl devices exposed to 78\%RH over 48 hours. Low-angle XRD spectra of (b) Au-hexyl and (d) Au-butyl devices exposed to humidity. Both hexyl- and butyl-MAPI show low- $n$ peaks below $2 \theta=12^{\circ}$ formed during fabrication, but these peaks do not grow with exposure time. 

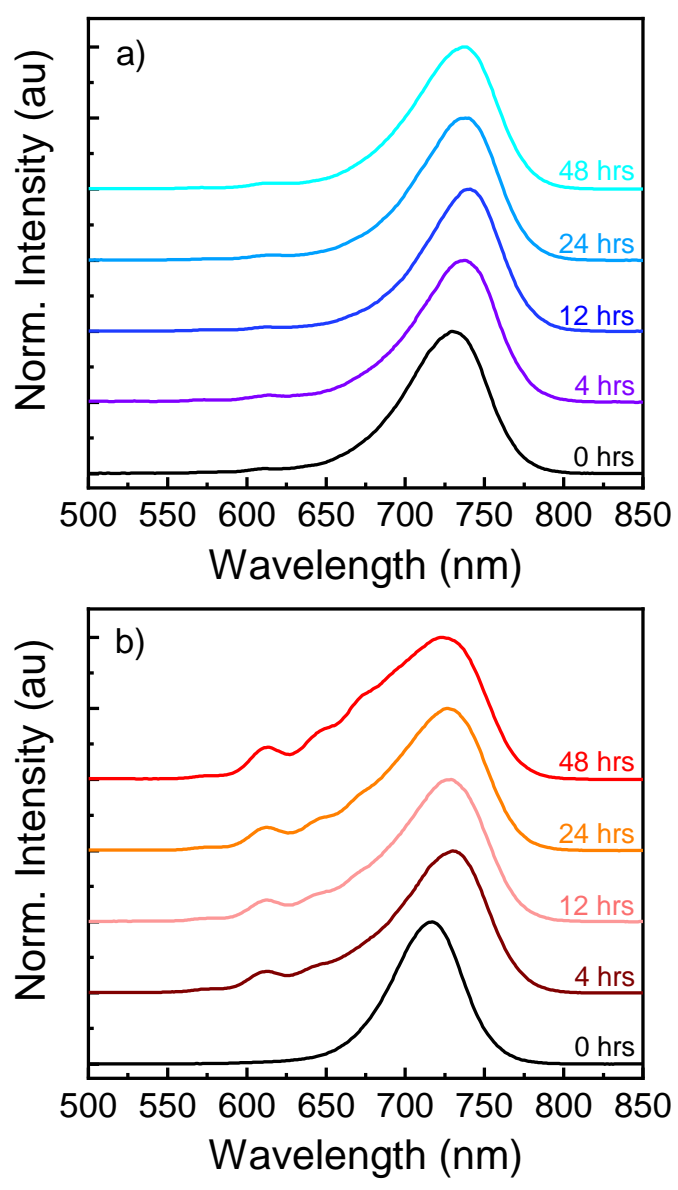

Figure S7 Normalized PL spectra of (a) hexylMAPI and (b) butyl-MAPI films over 48 hours of exposure to $78 \%$ RH. Both the hexyl- and butylMAPI films shows a slight $(\sim 10 \mathrm{~nm})$ red-shift of the primary peak, but the butyl-MAPI film also shows clear formation of low- $n$ peaks from $700-$ $600 \mathrm{~nm}$. 

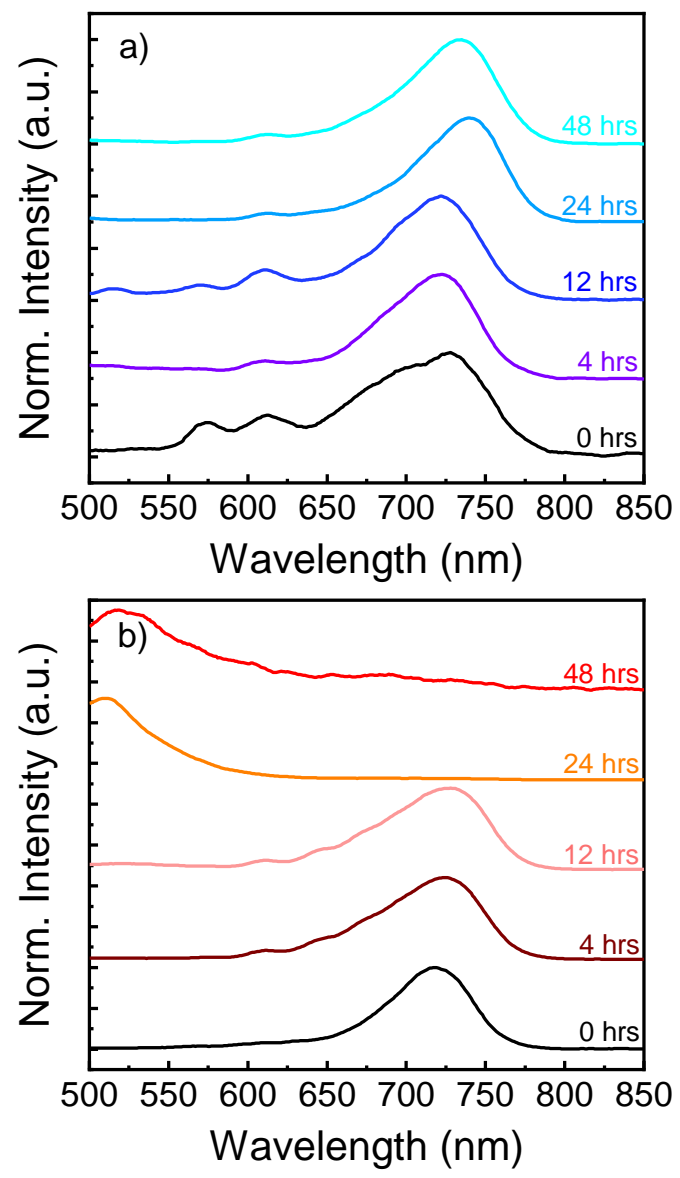

Figure S8 Normalized PL spectra of (a) Ag-hexyl and (b) Ag-butyl devices over 48 hours of exposure to $78 \% \mathrm{RH}$. Both the hexyl- and butylMAPI devices shows a slight $(\sim 10 \mathrm{~nm})$ red-shift of the primary peak, but the butyl-MAPI film also shows clear formation of low- $n$ peaks from 700 $550 \mathrm{~nm}$ up to 12 hours. Afterwards, the film shows only a broad emission below $550 \mathrm{~nm}$. The hexylMAPI film showed a decrease in the relative intensity of low- $n$ peaks with exposure with time. 

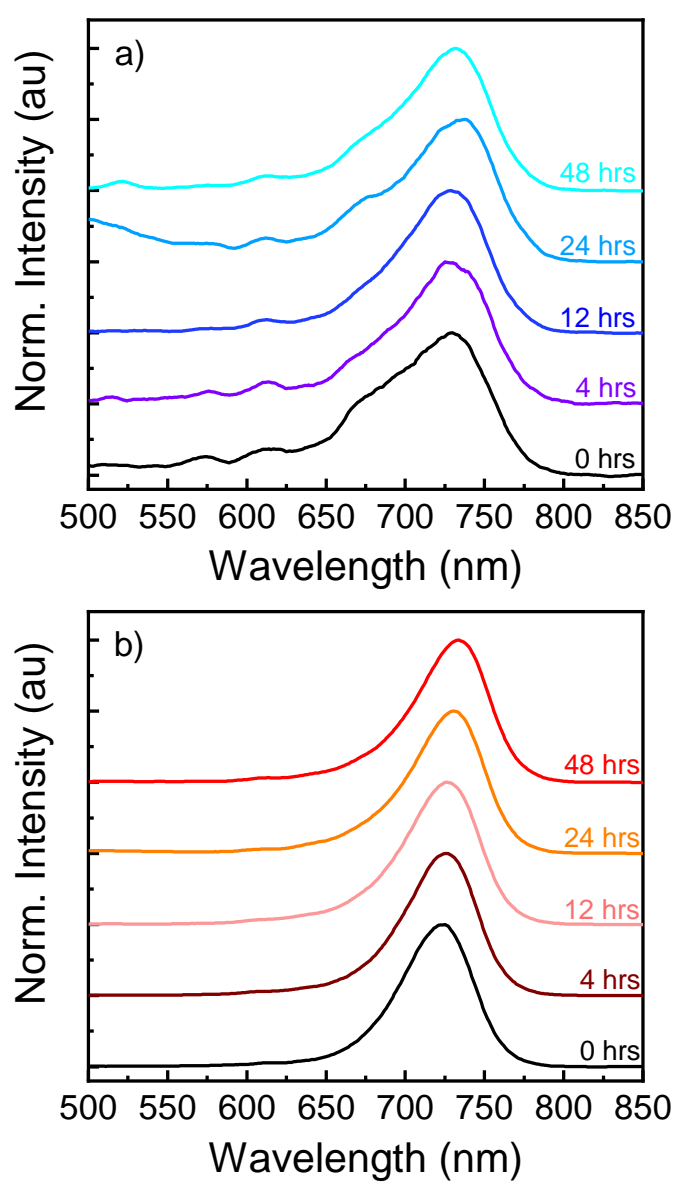

Figure S9 Normalized PL spectra of (a) Au-hexyl and (b) Au-butyl devices over 48 hours of exposure to $78 \%$ RH. Both the hexyl- and butylMAPI devices shows a slight $(\sim 10 \mathrm{~nm})$ red-shift of the primary peak, but the hexyl-MAPI device shows a decrease in the relative intensity of the low- $n$ peaks from $700-550 \mathrm{~nm}$. In contrast, the butyl-MAPI device shows almost no change. 

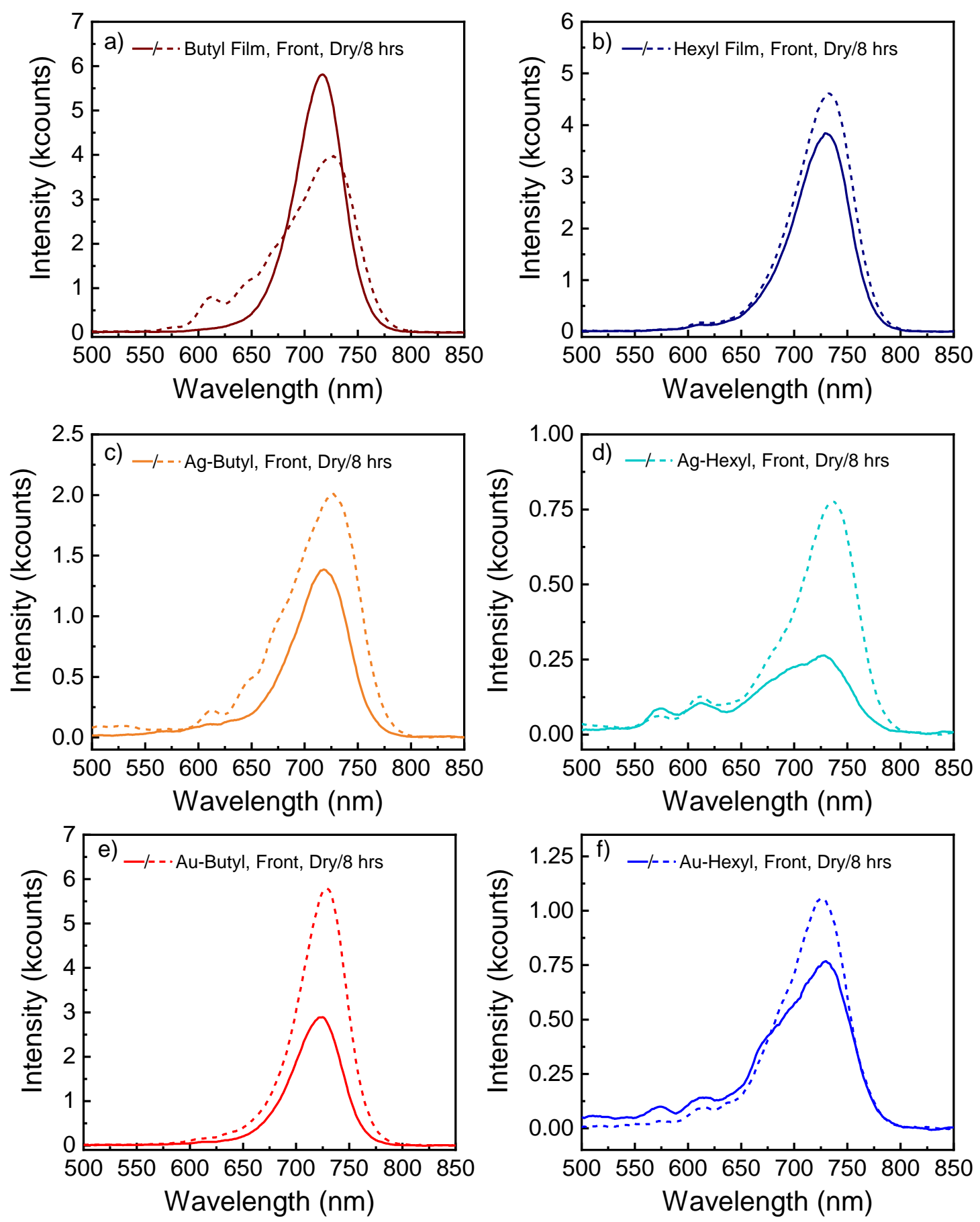

Figure S10 PL spectra of a (a) butyl-MAPI film, (b) hexyl-MAPI film, (c) Ag-butyl device, (d) Aghexyl device, (e) Au-butyl device, and (f) Au-hexyl device before and after exposure to $78 \%$ RH for 8 hours. The hexyl-MAPI film shows a slight increase in the $n>5$ peak with exposure, likely due to passivation of the surface, while the butyl-MAPI film shows a decrease in the primary $n>5$ peak and an increase in the low- $n$ peaks below $700 \mathrm{~nm}$, likely due to disproportionation of the perovskite. All of the devices show an increase in PL intensity with moisture exposure, indicating decreased quenching of carriers by the $\mathrm{C}_{60}$ due to the formation of a low- $n$ passivation layer at the surface of the film. Low- $n$ features are observed prior to degradation in (d) and (f), but do not appear to grow larger following degradation. 


\section{Butyl-MAPI}
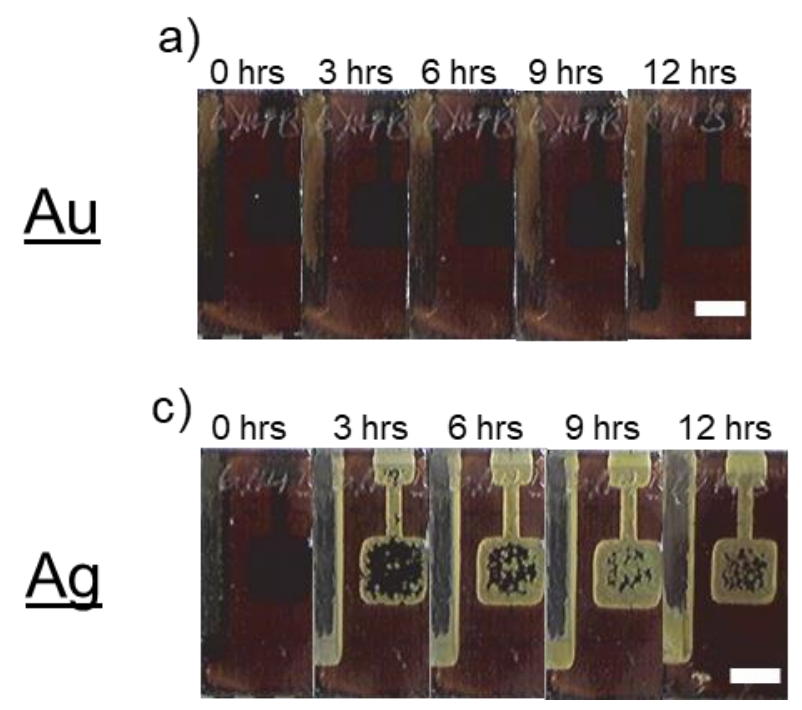

\section{$\underline{\text { Hexyl-MAPI }}$}

b)

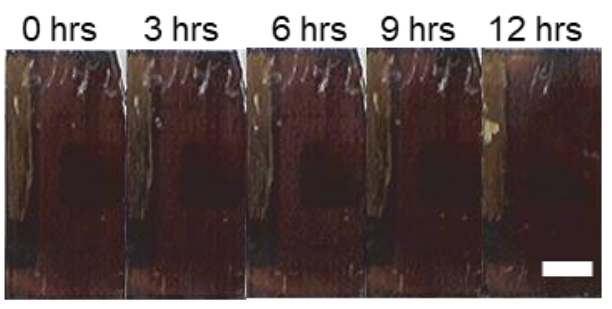

d)

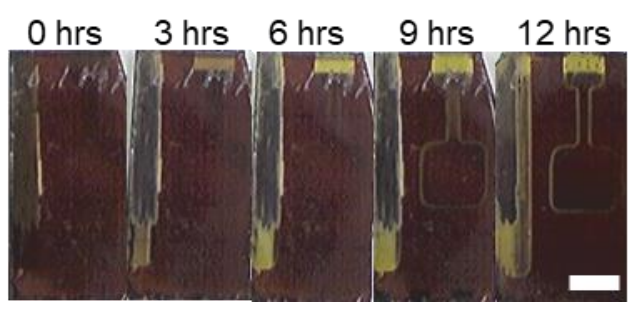

Figure S11 Photographs of the backs of (a) Au-butyl, (b) Au-hexyl, (c) Ag-butyl, and (d) Ag-hexyl devices over the course of 12 hours of exposure to $78 \% \mathrm{RH}$. Both (a) and (b) show little change to the devices over the time period, while (d) shows the formation of a thin strip of yellow material around the outer edges of the Ag contacts only. (c) shows the formation of a large quantity of yellow material around the outer edges of the contacts, as well as some areas in the interior after just 3 hours. The degradation continues over the next 9 hours, until almost the entire device is yellow. Of interest, the yellow material only forms in areas directly under or adjacent to the $\mathrm{Ag}$ electrode. The scale bar indicates $0.5 \mathrm{~cm}$. 

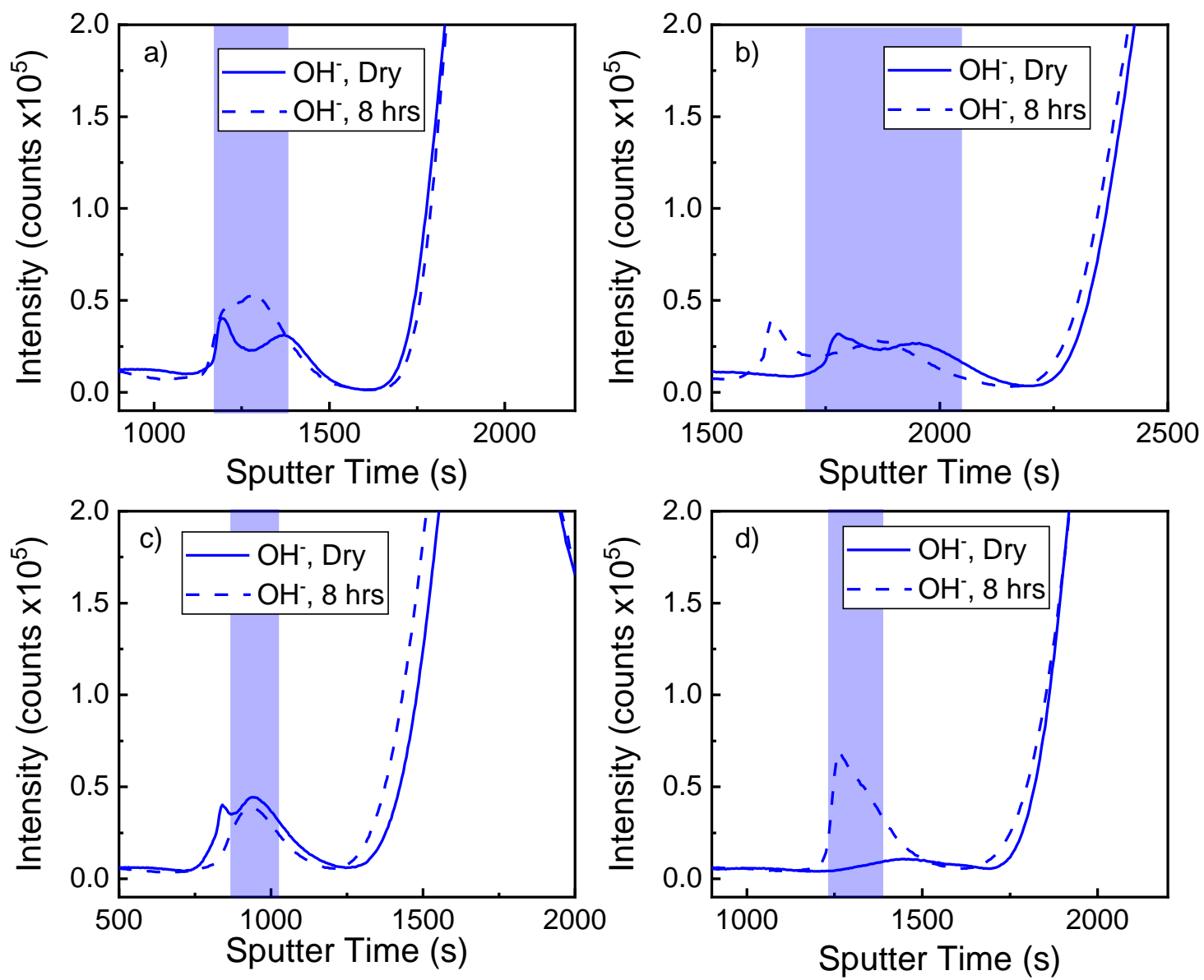

Figure $\mathbf{S 1 2} \mathrm{OH}^{-}$depth profiles of (a) Ag-butyl, (b) Au-butyl, (c) Ag-hexyl, and (d) Au-hexyl devices before and after 8 hours of exposure to $78 \% \mathrm{RH}$. The hydration region for each device is defined as the FWHM of the $\mathrm{OH}^{-}$peak at the $\mathrm{C}_{60}$ /perovskite interface following exposure. 

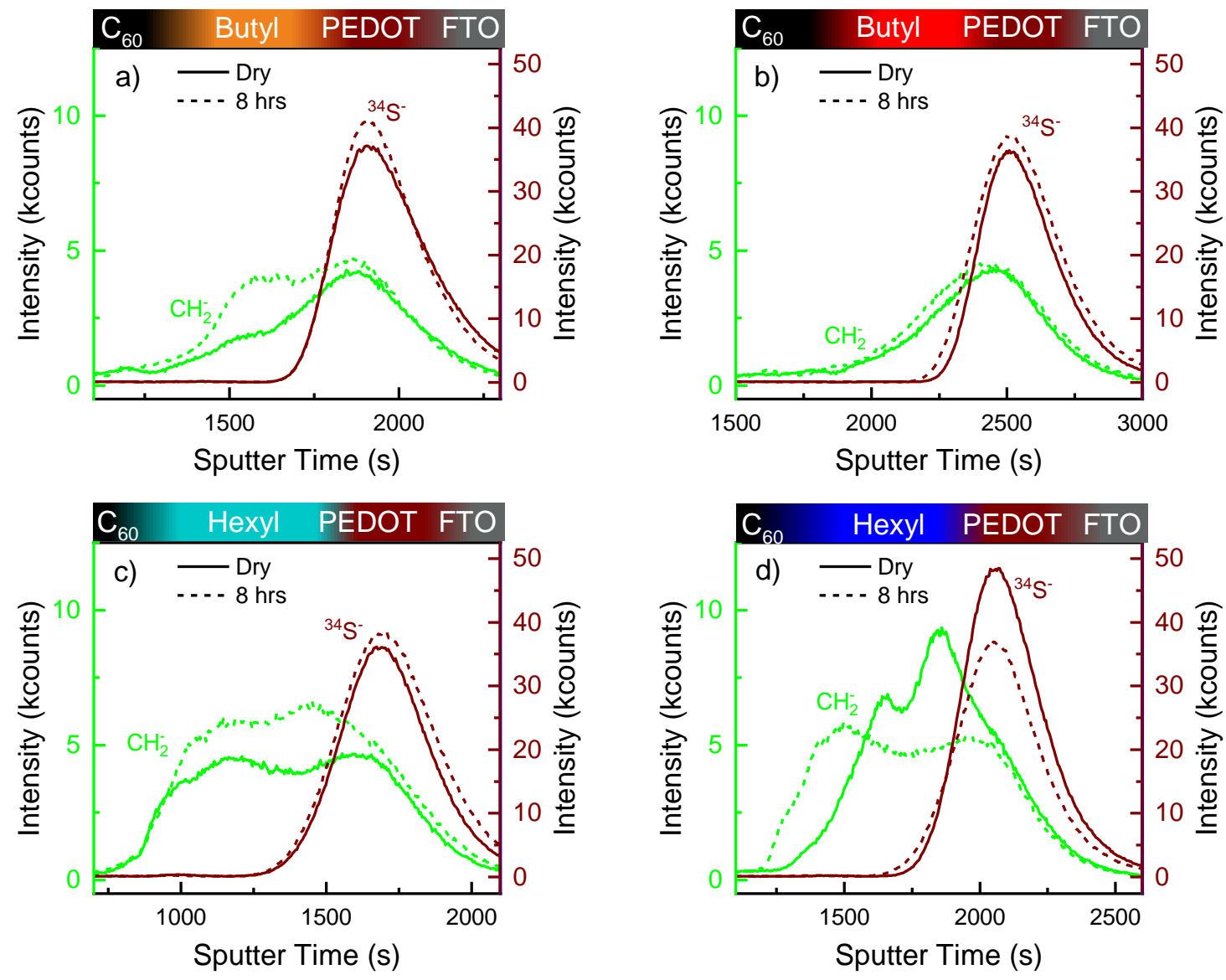

Figure $\mathrm{S13}_{2} \mathrm{CH}_{2}^{-}$and ${ }^{34} \mathrm{~S}^{-}$depth profiles for (a) Ag-butyl, (b) Au-butyl, (c) Ag-hexyl, and (d) Au-hexyl devices before and after moisture exposure. The colored bars on top of each profile show the relative location of the peaks within the larger device. In all cases, there is a $\mathrm{CH}_{2}^{-}$peak on the bottom (rightward) side of the perovskite layer that corresponds with the organics in the PEDOT (indicated by the ${ }^{34} \mathrm{~S}^{-}$species). 


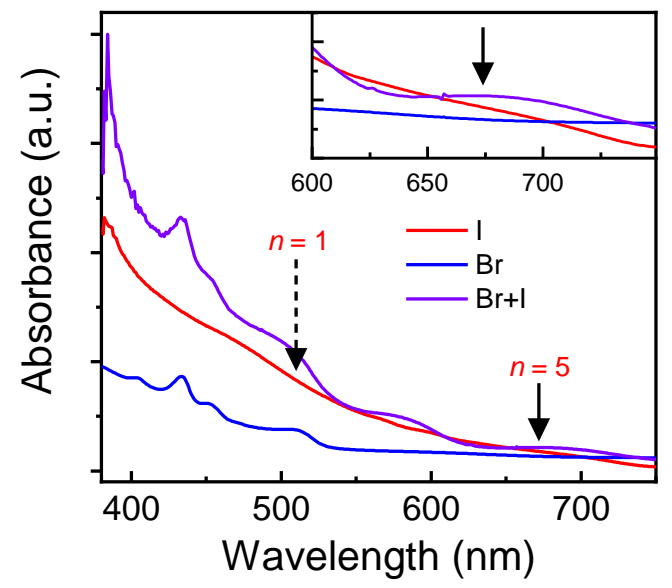

Figure S14 UV-Vis spectra of $n=5$ films of butylMAPI (red), butyl-MAPBr (blue), and a paired butyl iodo/bromo set (purple). The iodo $n=5$ band gap (arrow in inset) is poorly defined in the paired sample, as is the $n=1$ feature. 

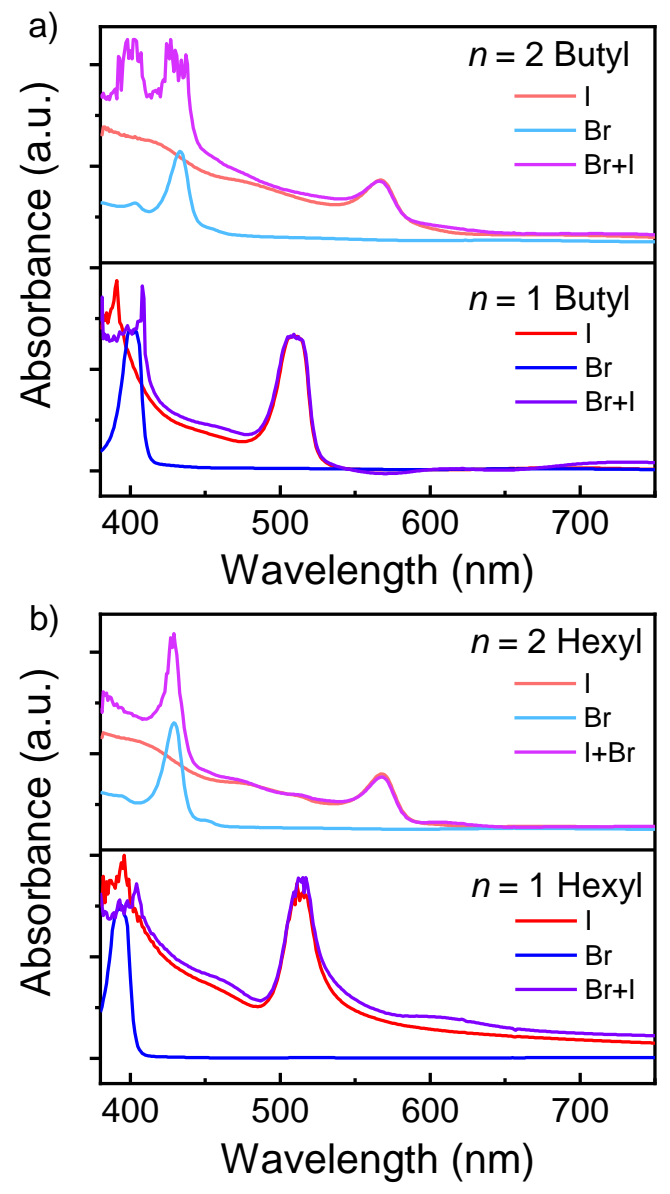

Figure S15 UV-Vis spectra of $n=2$ (top) and 1 (bottom) films of (a) butyl-MAPI and (b) hexylMAPI. The spectra include single iodo (red) and bromo (blue) materials, as well as a paired set of iodo/bromo films (purple). 

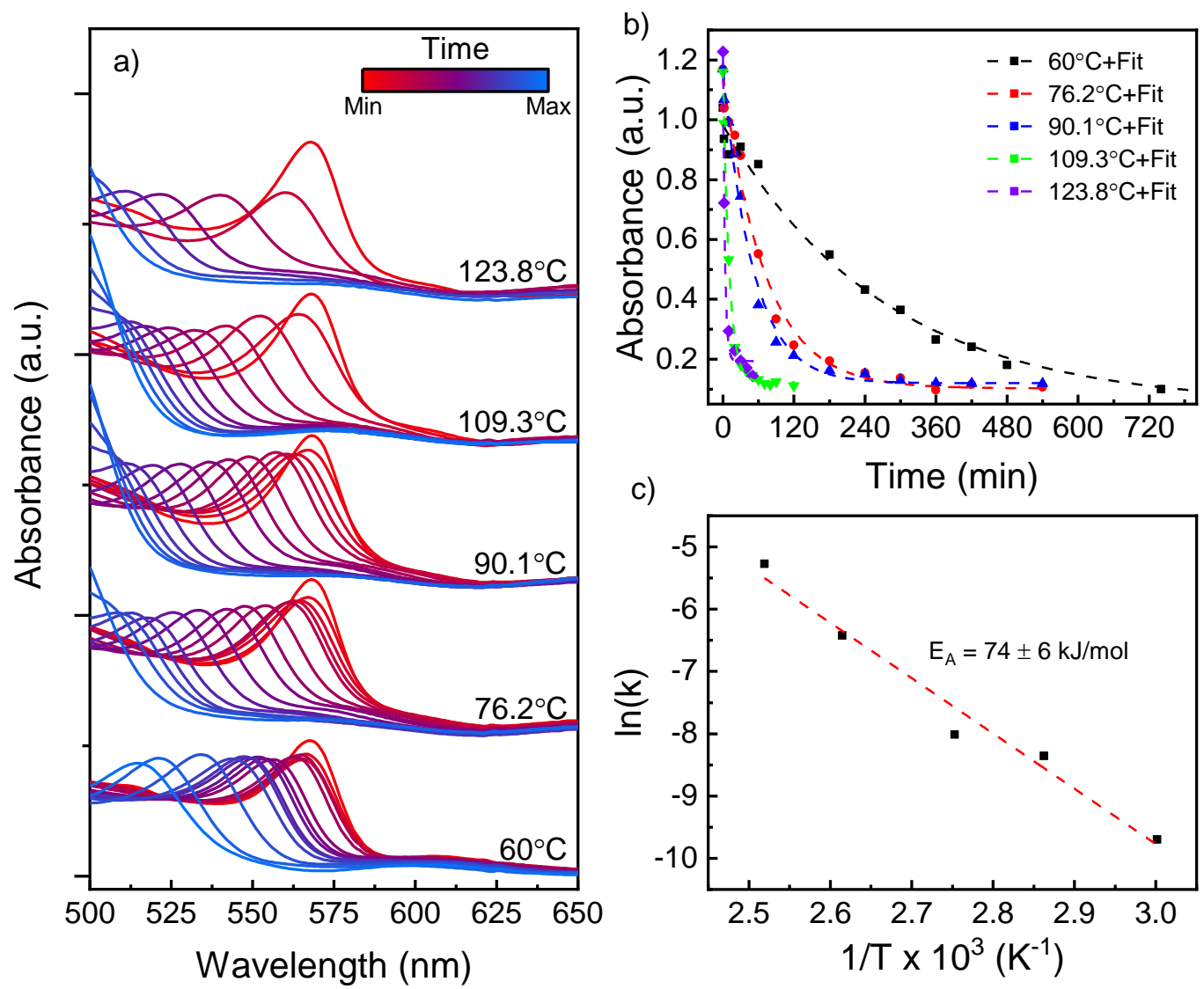

c)

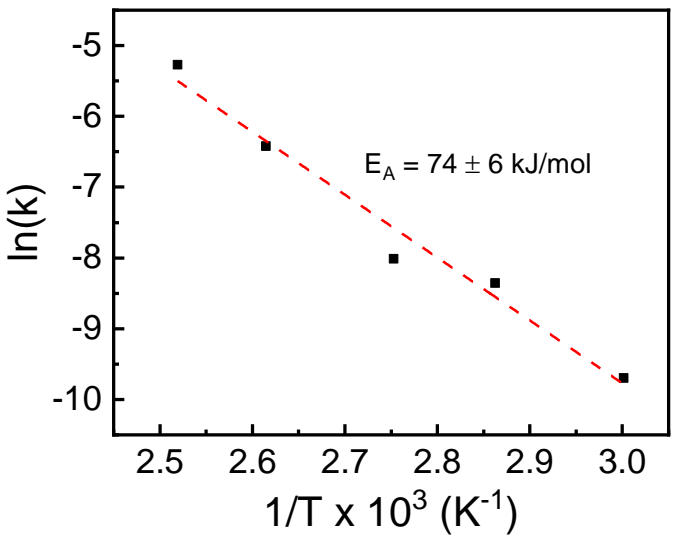

Figure S16 a) UV-Vis spectra of $n=2$ hexyl-MAPI iodo/bromo pairs held at several temperatures over time. b) Decay in the intensity of the iodo UV-Vis peak near $575 \mathrm{~nm}$ in (a) measured over time; each point corresponds to a spectrum in (a). The data is fit to first order decay (line). c) Arrhenius plot derived from decay constants calculated using the data in (b), providing the activation energy of halide migration in the material. 

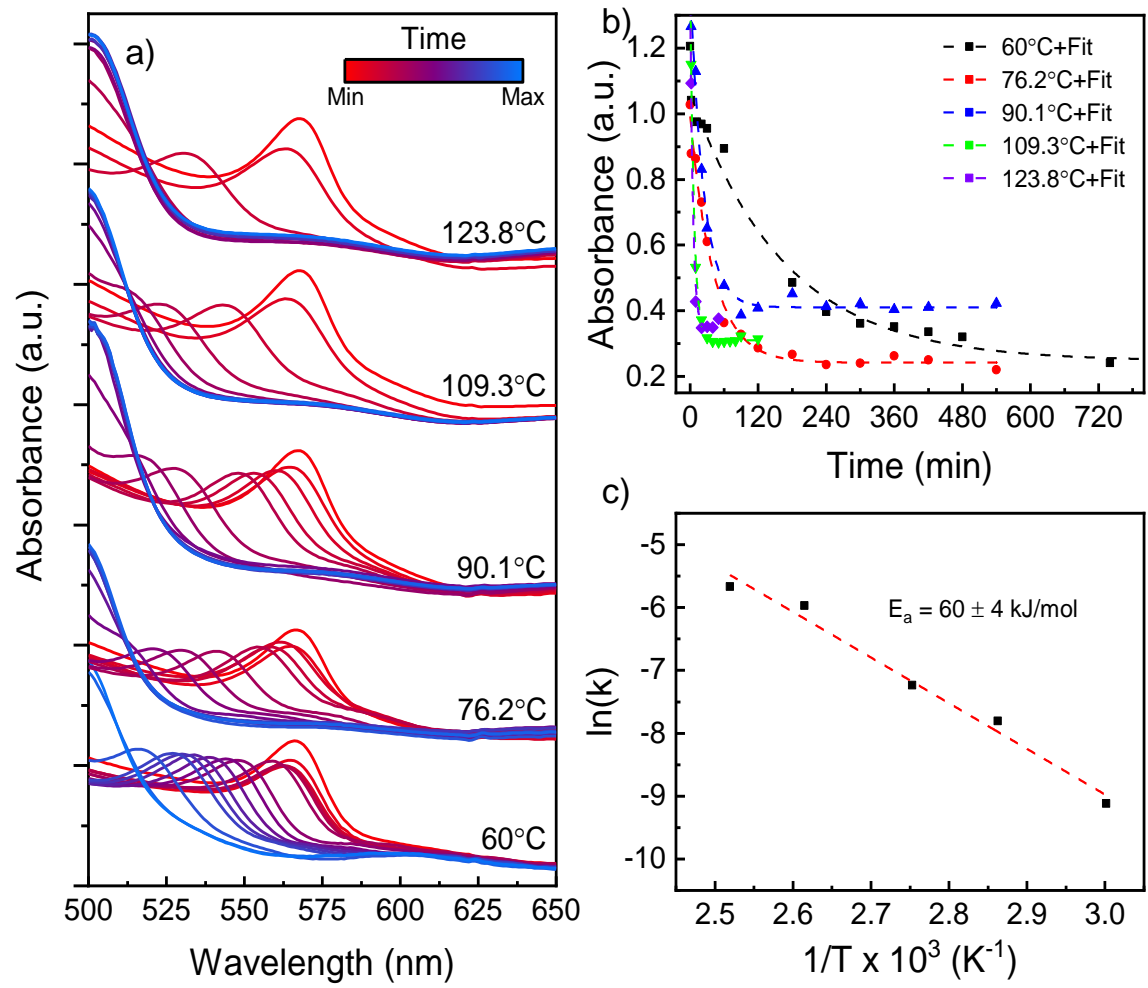

C)

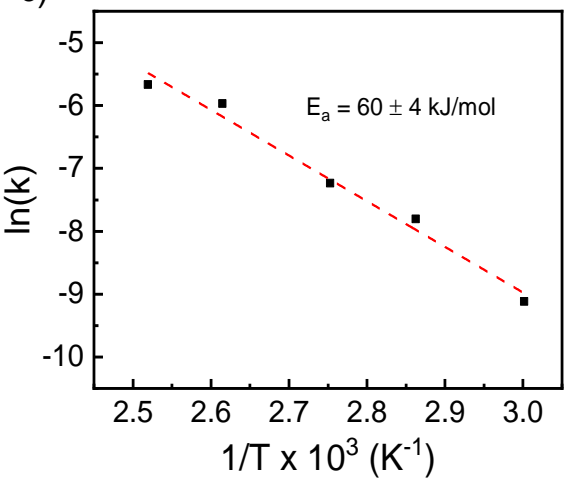

Figure S17 a) UV-Vis spectra of $n=2$ butyl-MAPI iodo/bromo pairs held at several temperatures over time. b) Decay in the intensity of the iodo UV-Vis peak near $575 \mathrm{~nm}$ in (a) measured over time; each point corresponds to a spectrum in (a). The data is fit to first order decay (line). c) Arrhenius plot derived from decay constants calculated using the data in (b), providing the activation energy of halide migration in the material. 

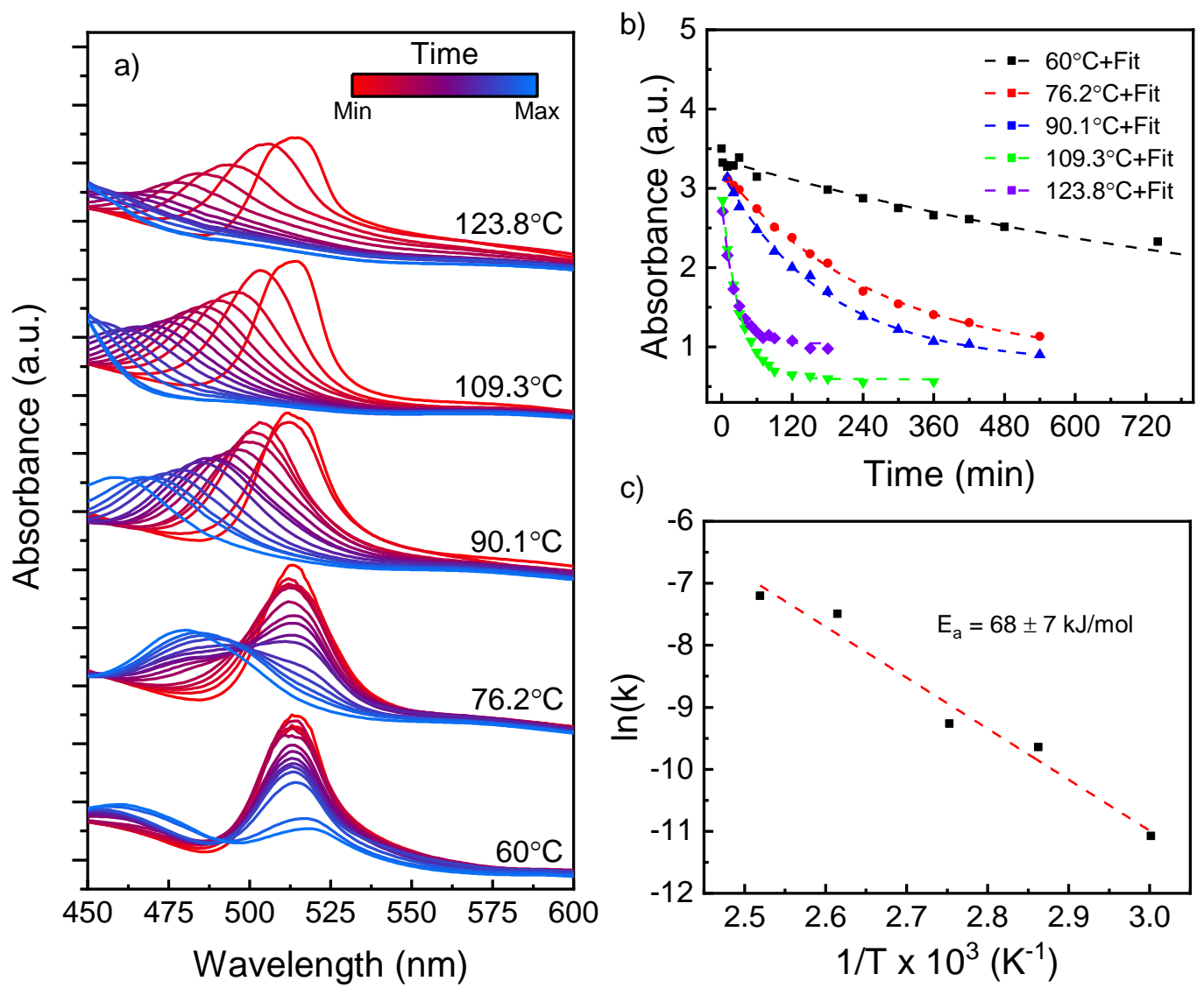

Figure S18 a) UV-Vis spectra of $n=1$ hexyl-MAPI iodo/bromo pairs held at several temperatures over time. b) Decay in the intensity of the iodo UV-Vis peak near $510 \mathrm{~nm}$ in (a) measured over time; each point corresponds to a spectrum in (a). The data is fit to first order decay (line). c) Arrhenius plot derived from decay constants calculated using the data in (b), providing the activation energy of halide migration in the material. 

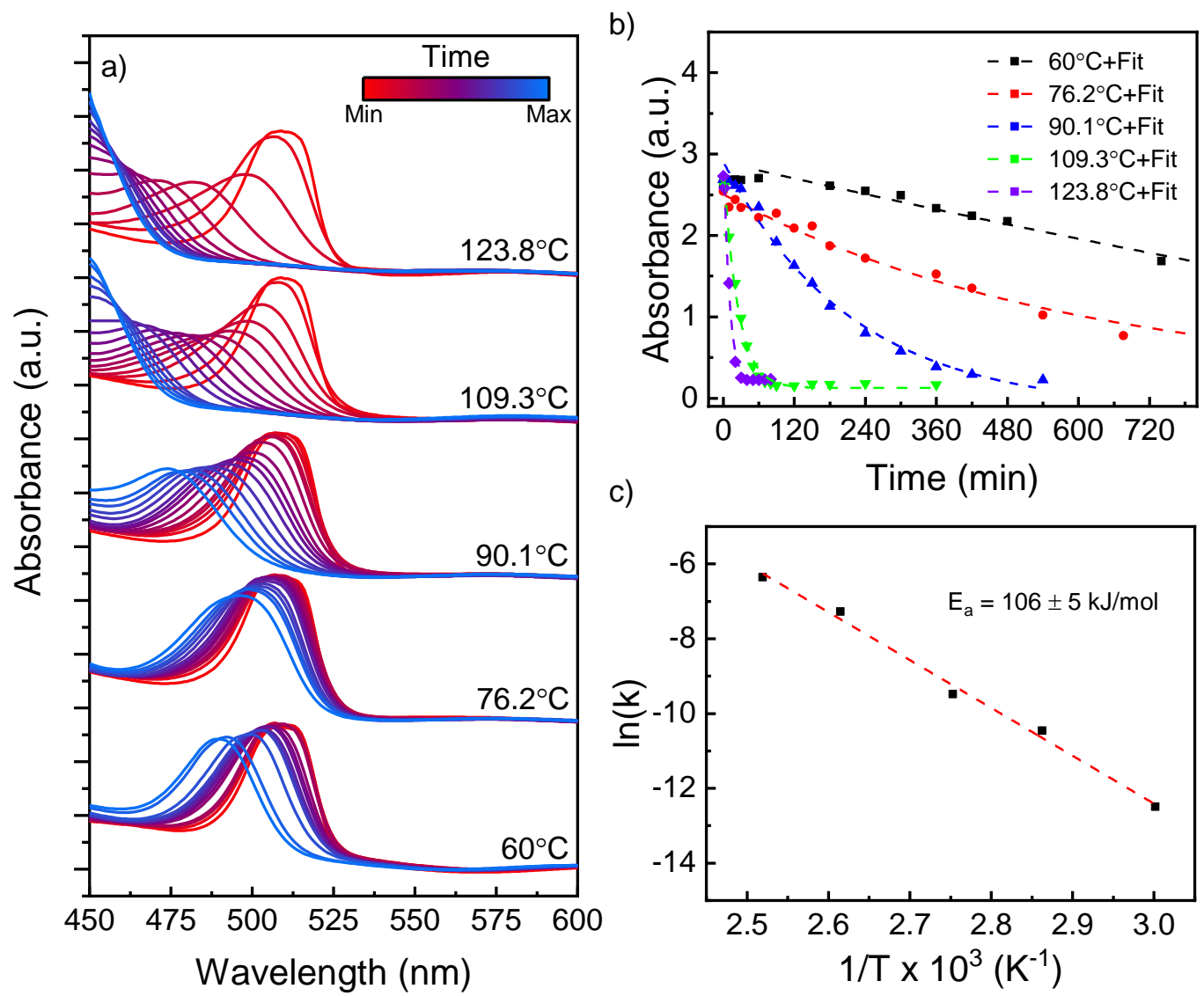

c)

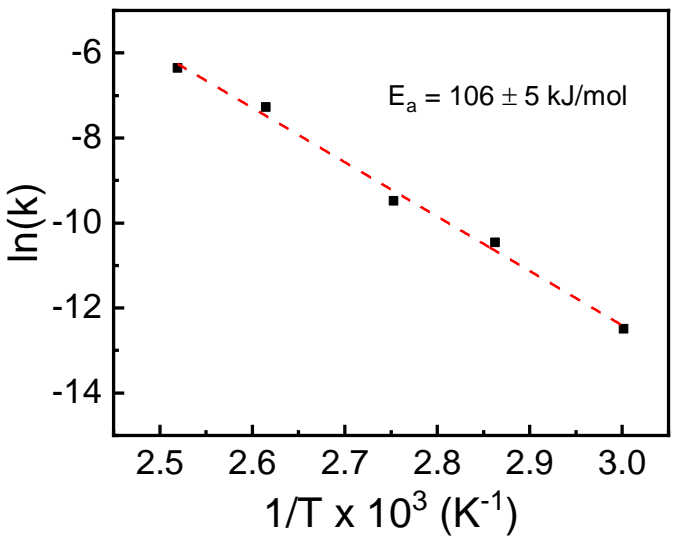

Figure S19 a) UV-Vis spectra of $n=1$ butyl-MAPI iodo/bromo pairs held at several temperatures over time. b) Decay in the intensity of the iodo UV-Vis peak near $510 \mathrm{~nm}$ in (a) measured over time; each point corresponds to a spectrum in (a). The data is fit to first order decay (line). c) Arrhenius plot derived from decay constants calculated using the data in (b), providing the activation energy of halide migration in the material. 


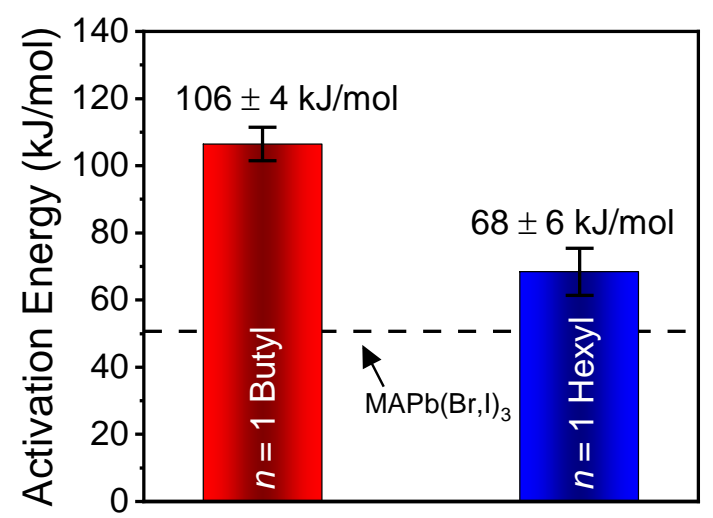

Figure S20 Activation energies for halide mobility in $n$ $=1$ butyl- and hexyl-MAPI perovskite determined by UV-Vis spectroscopy. The dashed line represents the previously reported activation energy of a 3D perovskite. 